\section{Pruni Cortex Extract Accelerates Wound Healing in a Mouse Model of Atopic Dermatitis}

\section{Mayumi Nomoto}

Department of Clinical Pharmacology, Faculty of Pharmaceutical Sciences, Niigata University of Pharmacy and Applied Life Sciences, 265-1 Higashijima, Akiha-ku, Niigata, Japan

*Corresponding author: Mayumi Nomoto, Department of Clinical Pharmacology, Faculty of Pharmaceutical Sciences, Niigata University of Pharmacy and Applied Life Sciences, 265-1 Higashijima, Akiha-ku, Niigata, Japan, Tel: (+81) 25-247-8800; Fax: (+81) 25-247-8810; E-mail: n.nomoto@beautifulskin.jp

Received date: October 10, 2017; Accepted date: October 27, 2017; Published date: November 03, 2017

Copyright: ( 2017 Nomoto M. This is an open-access article distributed under the terms of the Creative Commons Attribution License, which permits unrestricted use, distribution, and reproduction in any medium, provided the original author and source are credited.

\begin{abstract}
The crude drug Pruni Cortex (PC) is a component of Jumihaidokuto, which is a Chinese herbal medicine used for the treatment of skin diseases including atopic dermatitis (AD). To understand the role of PC in the mechanism of action of Jumihaidokuto on the skin, PC was administered to AD model mice. NC/Nga mice were induced to exhibit AD lesions by treating them with Dermatophagoides farina extract for 2 weeks and PC was orally administered for a further 4 weeks. The dermatitis score and histopathologic parameters were observed from 1 week after $A D$ induction (the acute phase of $A D$ ) to 4 weeks after $A D$ induction (the chronic phase of $A D$ ). At 4 weeks, the dermatitis score was significantly lower in the PC-treated group than in the untreated group. However, in the histopathological analysis conducted 1 week after AD induction, the PC-treated mice did not show fewer inflammatory cells in the dermis, but showed more fibroblasts in comparison with the untreated mice. At 4 weeks after AD induction, the PCtreated mice showed fewer inflammatory cells and fibroblasts than the untreated mice. PC may accelerate wound healing by increasing the number of fibroblasts in the dermis from the acute phase of atopic dermatitis.
\end{abstract}

Keywords: Pruni cortex; Atopic dermatitis; Fibroblast; Inflammation

\section{Introduction}

Pruni Cortex (PC) is obtained from Prunus jamasakura or other related plants in the family Rosaceae as a crude drug extracted from bark without the periderm [1]. Since ancient times, it has been used widely in Japanese folk medicine, including for detoxification, the treatment of eczema, and as an antitussive [2]. It is also included in Jumihaidokuto, which is a Chinese herbal medicine that has been applied for the treatment of various skin diseases including acne vulgaris and atopic dermatitis (AD) [3]. The pharmacological actions of PC include antibacterial and antioxidant activities, the stimulation of estrogen secretion, and the inhibition of sebum synthesis and neutrophil activity; therefore, PC is expected to have various indications for skin diseases [1,4-7].

To elucidate the mechanism of action of PC against AD, PC extract $(1 \mathrm{~g} / \mathrm{kg})$ was orally administered to mite antigen-sensitized AD model mice for 2 weeks and the severities of the skin findings and histopathological changes were evaluated [8]. The PC-treated group showed a significant improvement in the severity of skin findings and a reduction in the infiltration of skin tissue inflammatory cells in comparison with the untreated group (Figure 1). However, immunostaining for TE7, a fibroblast-specific cell surface marker, resulted in no obvious changes in comparison with the untreated group. After the administration of PC for 2 weeks, exterior images and hematoxylin-eosin staining revealed an improvement in the severity of AD. However, no change was seen in the number of dermal fibroblasts. We considered that PC may have influenced the process of wound healing and therefore decided to focus on the temporal changes through repeated observations of histopathologic parameters from the

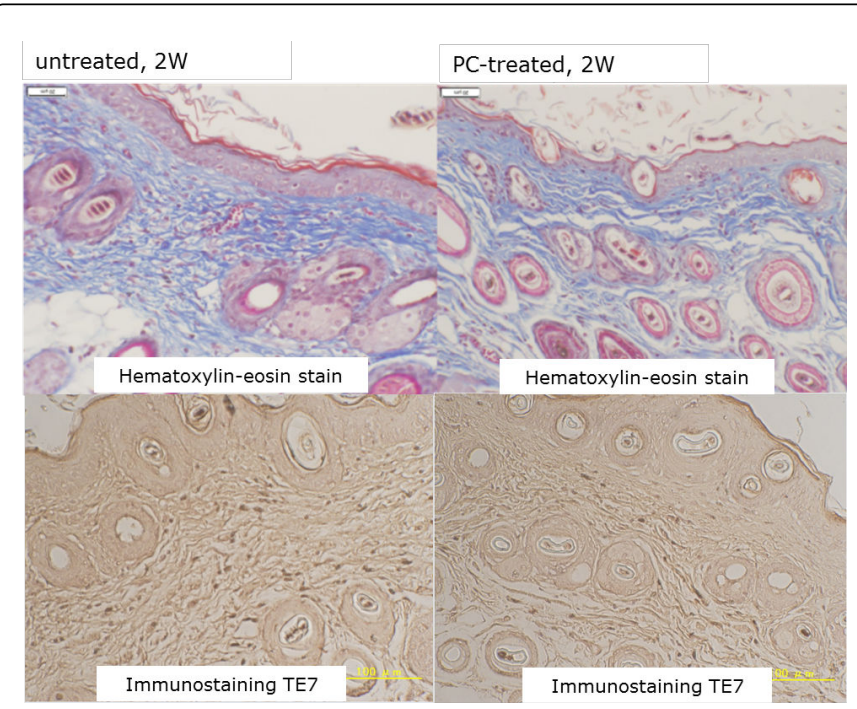

Figure 1: Skin tissue 2 weeks after PC administration after hematoxylin-eosin staining (top) and immunostaining (below). Left: untreated; right: PC-treated. A band in the upper part of each image is epidermis and the blue part is the dermis.

\section{Materials and Methods}

The experimental method was in accordance with that described by Watanabe et al. [8]. 


\section{Materials}

Biostir-AD, which is a cream formulation containing the extract of Dermatophagoides farina (DfE, commonly known as the house dust mite) was purchased from Biostir (Kobe, Japan). Phosphatase arrest-III was purchased from G-Biosciences (St. Louis, MO, USA). Bovine serum albumin (BSA), 2-mercaptoethanol, sodium chloride, sodium fluoride, sodium orthovanadate, Trizma ${ }^{\circ}$ base, and Tween ${ }^{\circ} 20$ were purchased from Wako Pure Chemical Industries (Osaka, Japan). Naringenin and genistein were purchased from LKT Laboratories (Phalen Boulevard, St. Paul, MN, USA). Sakuranetin was purchased from Extrasynthese (Genay Cedex, France). Unless otherwise stated, all other reagents were of analytical grade and were purchased from Sigma-Aldrich (St. Louis, MO, USA).

\section{Preparation of the PC extract}

The PC extract was gifted from Kracie Pharma (Kampo Research Laboratories, Toyama, Japan). The cut crude drug of PC (1.5 kg) was soaked in $15 \mathrm{~L}$ water, boiled for $1 \mathrm{~h}$, and then centrifuged to remove the residue. The extract was further concentrated by spray drying and the final crude extract $(180 \mathrm{~g})$ was stored at $4^{\circ} \mathrm{C}$ until use.

\section{Preparation of standard and sample solutions for high- performance liquid chromatography (HPLC)}

Stock solutions $(1,000 \mu \mathrm{g} / \mathrm{mL})$ of genistein, naringenin, and sakuranetin were prepared immediately before use and used as reference standards. The hot water extract of PC was diluted in water to $2 \mathrm{mg} / \mathrm{mL}$ and used as the sample solution. The diluted PC extract was filtered through a disposable $0.45-\mu \mathrm{m}$ polyvinylidene fluoride syringe filter (EMD Millipore, Bedford, MA, USA) and directly injected.

\section{HPLC fingerprinting of the PC extract}

The components of the PC extract were analyzed using an HPLC system comprised of a 10AD liquid chromatograph and a DGU-20A3R degasser (Shimadzu, Kyoto, Japan). An isocratic reverse-phase chromatographic analysis was performed using a Shim-pack CLC column (CLC-ODS(M), $250 \times 4.6 \mathrm{~mm}$ internal diameter; Shimadzu) with a particle size of $5 \mu \mathrm{m}$ at $26^{\circ} \mathrm{C}$. An injection volume of $20 \mu \mathrm{L}$, a flow rate of $1 \mathrm{~mL} / \mathrm{min}$, and a detection wavelength of $280 \mathrm{~nm}$ were used. For the analysis of naringenin and genistein, the mobile phase comprised phosphate buffer ( $\mathrm{pH} 7$ ) and acetonitrile (70:30). For the analysis of sakuranetin, the mobile phase comprised methanol and water $(6: 4)[9,10]$.

In each analysis, the identities of the resulting chromatographic peaks were confirmed by comparing their retention times and ultraviolet-visible spectra with those of the reference standards. The standard solutions of genistein, naringenin, and sakuranetin were injected into the HPLC at various dilutions $(1-100 \mu \mathrm{g} / \mathrm{mL})$ and the peak height responses were obtained. Standard curves were prepared by plotting concentration against peak height. The quantification was performed by integrating the peak heights of the samples using the corresponding standard curve.

\section{Animals}

Specific-pathogen-free female 6-week-old NC/Nga mice were purchased from Charles River Japan (Yokohama, Japan). They were housed in a controlled room $\left(23 \pm 2^{\circ} \mathrm{C}, 12\right.$-h light cycle). After 1 week, the mice were randomly divided into three groups: an untreated group (normal, $\mathrm{n}=6)$ and DfE cream-treated mice (100 mg/mouse) that received either vehicle ( $1 \%$ methyl cellulose) $(A D, n=6)$ or aqueous $P C$ extract $(1 \mathrm{~g} / \mathrm{kg} /$ day in the evening) $(\mathrm{AD}+\mathrm{PC}, \mathrm{n}=6)$ by oral administration. All three groups were provided with water and chow ad libitum for the entire duration of the study (4 weeks).

The mice were weighed weekly and food intake was estimated at the same time on every second day. The animal experiments were performed in accordance with national guidelines and approved by the animal care committee of Niigata University of Pharmacy and Applied Life sciences.

\section{AD induction in NC/Nga mice}

$\mathrm{NC} / \mathrm{Nga}$ mice were induced to exhibit skin lesions using DfE cream by a previously described method [11]. In brief, the hair on the upper back was shaved and the skin barrier was disrupted by applying $150 \mu \mathrm{L}$ $4 \%(\mathrm{w} / \mathrm{v})$ sodium dodecyl sulfate to the shaved dorsal skin and both surfaces of each ear. After $3 \mathrm{~h}$, DfE cream (100 mg) was applied topically. This procedure was performed twice per week for 2 weeks. PC aqueous extract $(1 \mathrm{~g} / \mathrm{kg} /$ day $)$ was administered orally in the evening at a dose ten times greater than the human dose used in clinical settings (6-9 kg/day/person). During our preliminary studies, we observed that this dose was effective and had no adverse effects.

PC treatment was initiated after the second week of $\mathrm{AD}$ induction and continued for 4 weeks (a total period of 6 weeks). The duration of the study was selected based on previous reports [12]. On the last day of the study period (the end of the 6th week), all the mice were weighed and sacrificed, and their skin tissue was harvested for immunohistochemical and semi-quantitative immunoblotting analyses.

\section{Evaluation of dermatitis severity}

The relative severity of dermatitis was assessed macroscopically each week by evaluating the area affected by eczema and the disease severity index according to the following scoring system: 0 , no symptoms; 1 , mild symptoms; 2, moderate symptoms; 3 , severe symptoms. The dermatitis score was defined as the sum of scores for erythema/ hemorrhage, excoriation/erosion, edema, and scaling/dryness [13].

\section{Histopathological analysis}

The excised skin was cut into approximately 2 -mm-thick transverse slices and fixed in $10 \%$ formalin. Sections of 3-5 $\mu \mathrm{m}$ thickness were stained with hematoxylin and eosin for histological analysis. Histomorphological evaluations of all the skin sections were carried out in a blinded manner.

\section{Immunohistochemical analysis}

The remaining skin sections were immunohistochemically stained for TE7. After being deparaffinized and hydrated, the slides were washed in Tris-buffered saline (TBS; $10 \mathrm{mM}$ Tris- $\mathrm{HCl}, 0.85 \% \mathrm{NaCl}$, $\mathrm{pH}$ 7.2). The activity of endogenous peroxidases was quenched by incubating the slides under $0.3 \% \mathrm{H}_{2} \mathrm{O}_{2}$ in methanol. The slides were blocked using either $10 \%$ goat serum or rabbit serum for $1 \mathrm{~h}$ at room temperature and washed three times with TBS for 5 min each.

After being incubated overnight with the primary antibody at $4^{\circ} \mathrm{C}$, the slides were washed in TBS and a horseradish peroxidase- 
conjugated secondary antibody was added and incubated at room temperature for a further $45 \mathrm{~min}$. The slides were rinsed in TBS, incubated with a colorimetric reagent (3,3'-diaminobenzidine tetrahydrochloride) for the detection of bound antibodies, and finally counterstained with hematoxylin.

\section{Statistical Analysis}

The data are presented as the mean \pm standard error of the mean. The significance of differences among means was analyzed using either one-way analysis of variance followed by Tukey's multiple comparison test or two-tailed t-test, as appropriate. Values of $\mathrm{p}<0.05$ were considered statistically significant. Prism 5 software (GraphPad Software, San Diego, CA, USA) was used for all statistical calculations.

\section{Results}

\section{HPLC analysis}

To confirm the major bioactive components of the PC extract, a 200 $\mu \mathrm{g} / \mathrm{mL}$ solution of the extract was subjected to HPLC fingerprinting and the peaks corresponding to the major bioactive components genistein, naringenin, and sakuranetin were identified from their ultraviolet-visible absorption spectra at $280 \mathrm{~nm}$. These values were confirmed through comparison with the reference standards (10 $\mu \mathrm{g} / \mathrm{mL}$ genistein, naringenin, or sakuranetin) either alone or mixed with a PC extract solution of a similar concentration. The amounts of genistein, naringenin, and sakuranetin in $\mathrm{PC}$ were found to be 1.15 , 4.68 , and $1.55 \mathrm{mg} / \mathrm{g}$, respectively (Figures $2 \mathrm{~A}-2 \mathrm{D}$ and $3 \mathrm{~A}-3 \mathrm{C}$ ).

\section{Effects of PC on skin histopathology}

At 1 week after $\mathrm{AD}$ induction, which is the acute phase of $\mathrm{AD}$, the skin tissues of the PC-treated mice did not show fewer inflammatory cells in the dermis, but showed more fibroblasts than those of the untreated mice (Figure 4). At 4 weeks after AD induction, which is the chronic phase of $\mathrm{AD}$, the tissues of the PC-treated mice showed fewer inflammatory cells and fibroblasts in the dermis than those of the untreated mice (Figure 5).

\section{Effects of PC extract on dermatitis severity scores}

Significantly lower dermatitis severity scores were observed at 1 and 4 weeks after $\mathrm{AD}$ induction in the PC-treated group in comparison with those in the untreated group (Figure 6).

\section{Discussion}

Fibroblasts are cells that are commonly found in connective tissue, produce dermal extracellular matrix components, such as collagen, elastin, and hyaluronic acid [14,15], and induce wound healing [16]. These cells maintain skin homeostasis by contributing to processes such as wound healing, and their involvement in the pathology of $\mathrm{AD}$ has also been suggested. An increased number of fibroblasts in the dermis of chronic AD lesions [17] and the induction of epidermal tissue structural defects [18] have been reported. It is considered that fibroblasts produce dermis components in the acute phase after wounding, which promotes wound healing. By contrast, an abnormal increase in the number of fibroblasts observed during the chronic phase of wound healing or a pathological condition may delay the healing process.
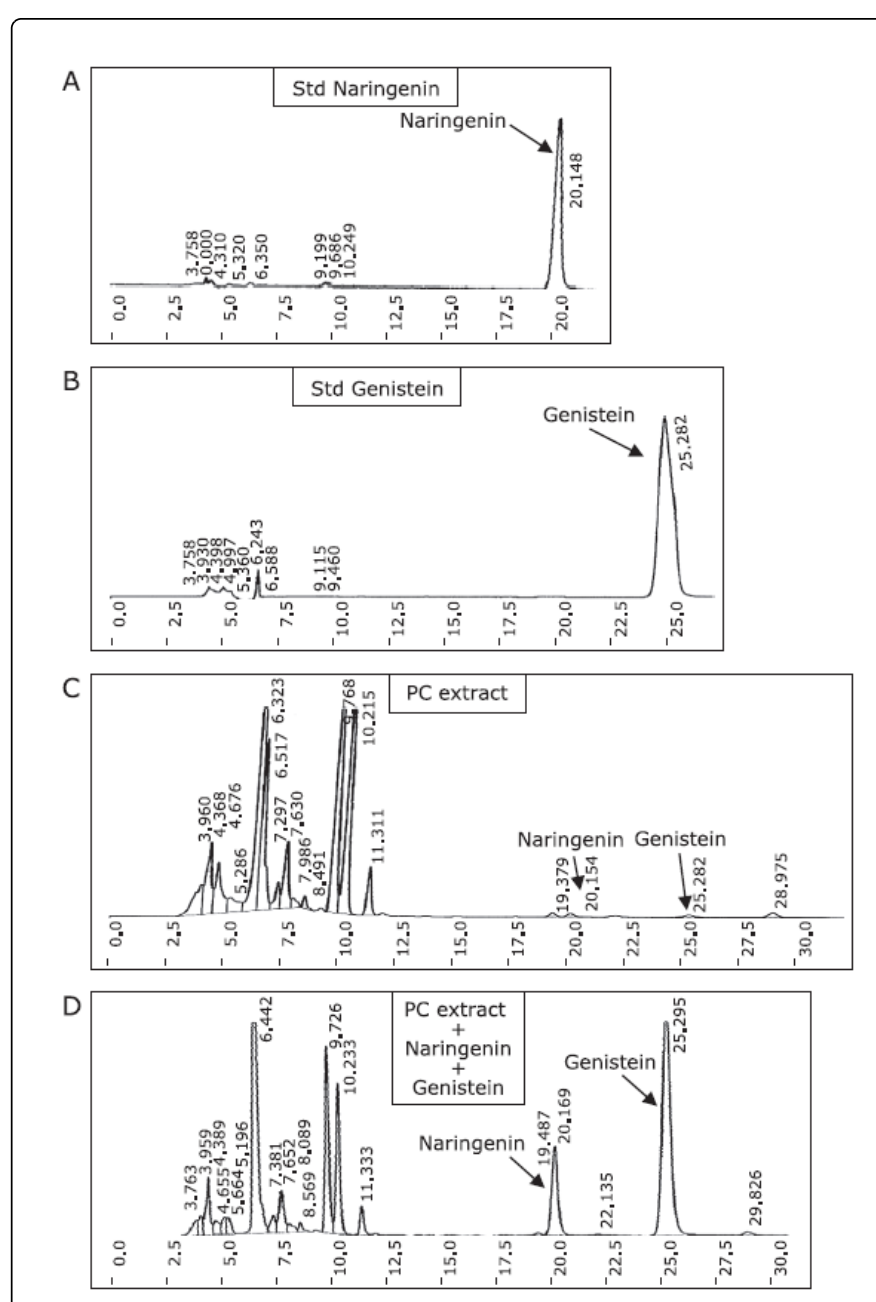

Figure 2: HPLC fingerprint of PC aqueous extract was obtained as described in the Methods section. (A), Standard naringenin $(10 \mu \mathrm{g} /$ $\mathrm{mL})$. (B), Standard genistein $(10 \mu \mathrm{g} / \mathrm{mL})$. (C), PC extract $(200 \mu \mathrm{g} /$ $\mathrm{mL})$. (D), PC extract with standard naringenin and genistein mixture (similar concentration). Reference standards of naringenin and genistein were used to compare the obtained peaks.

When PC extract was administered to AD mice, a histopathologic analysis at 1 week after $\mathrm{AD}$ induction, which is the acute phase of $\mathrm{AD}$, showed no decrease in the number of inflammatory cells in the dermis, but showed an increase in the number of fibroblasts. The number of fibroblasts, which are critical in the promotion of healing, increased from the first week of treatment, which suggested that PC accelerated the resolution of the inflammation in the skin affected by $\mathrm{AD}$ lesions (Figure 7). The stimulatory effect of PC on estrogen secretion may be related to this increase in the number of fibroblasts, since estrogen is known to activate fibroblasts through the estrogen receptor found on fibroblasts. It has been reported that PC binds to estrogen receptor $\beta$ in vitro and increases estrogen secretion from skin fibroblasts [1]. 
Citation: Nomoto M (2017) Pruni Cortex Extract Accelerates Wound Healing in a Mouse Model of Atopic Dermatitis. J Nutr Disorders Ther 7:

Page 4 of 5

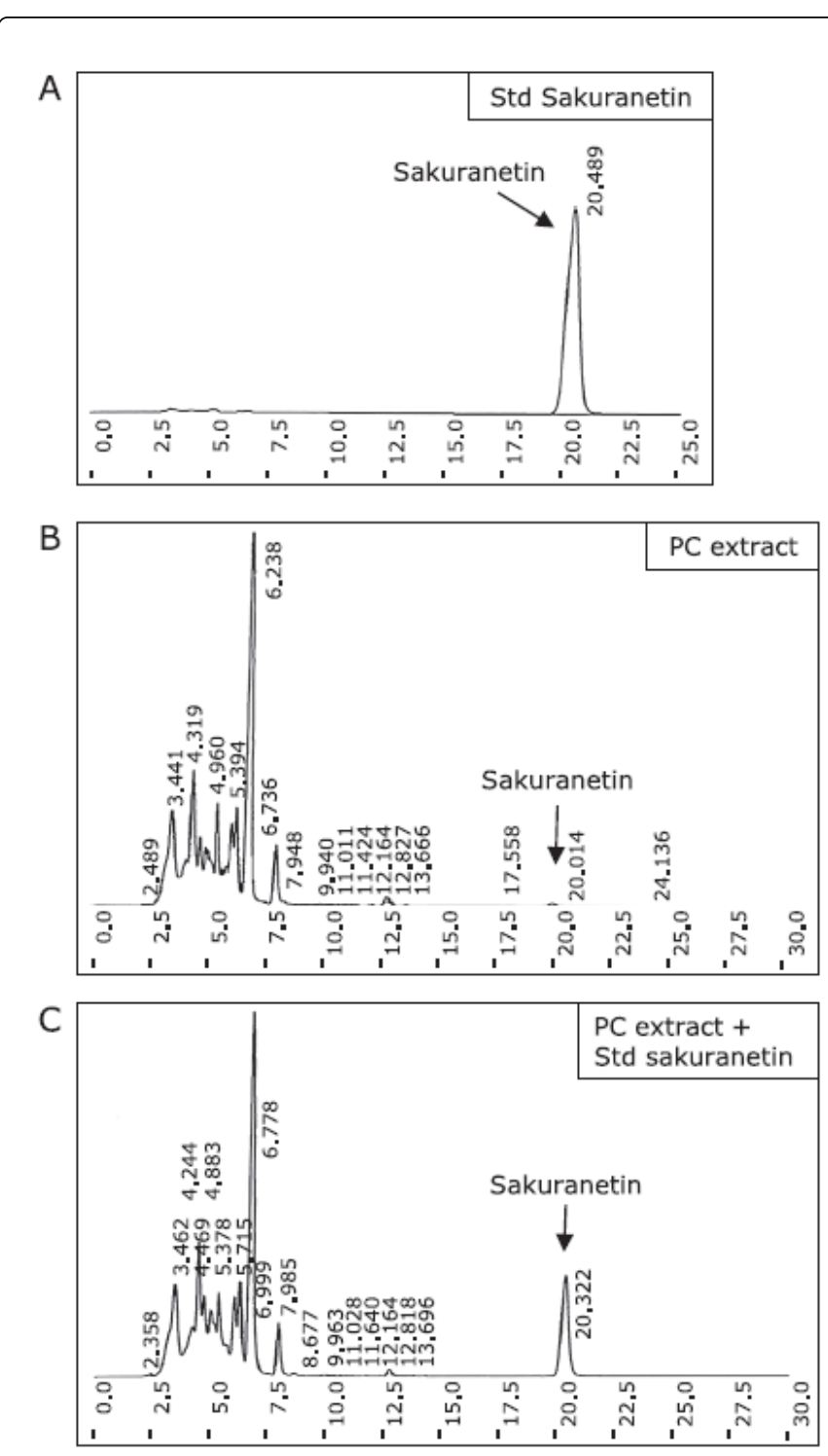

Figure 3: HPLC fingerprint of PC aqueous extract was obtained as described in the Methods section. (A), Standard sakuranetin $(10 \mu \mathrm{g} /$ $\mathrm{mL})$. (B), PC extract $(200 \mu \mathrm{g} / \mathrm{mL})$. (C), PC extract with standard sakuranetin mixture (at a similar concentration). Reference standards of sakuranetin were used to compare the obtained peaks.

At 4 weeks after $\mathrm{AD}$ induction, which is the chronic phase of $\mathrm{AD}$, the PC-treated mice showed a decrease in the number of inflammatory cells in the dermis and only a few fibroblasts were seen. The promoting effect of PC on the proliferation of fibroblasts can be observed during the acute phase when treatment is needed; however, this effect may not occur after the condition has improved.

Such physiologically normalizing effects are often observed during the clinical use of Chinese herbal medicines. For example, the medicine Kakkonto, which is usually prescribed for the common cold, is known to promote healing through the induction of fever, instead of reducing fever through the induction of chills [19]. Taken together, the observations of Chinese herbal medicines in clinical practice and experimental research have indicated that such medicines usually do not strongly inhibit or promote particular biological processes, but rather subtly accelerate the inherent wound healing ability of the individual.

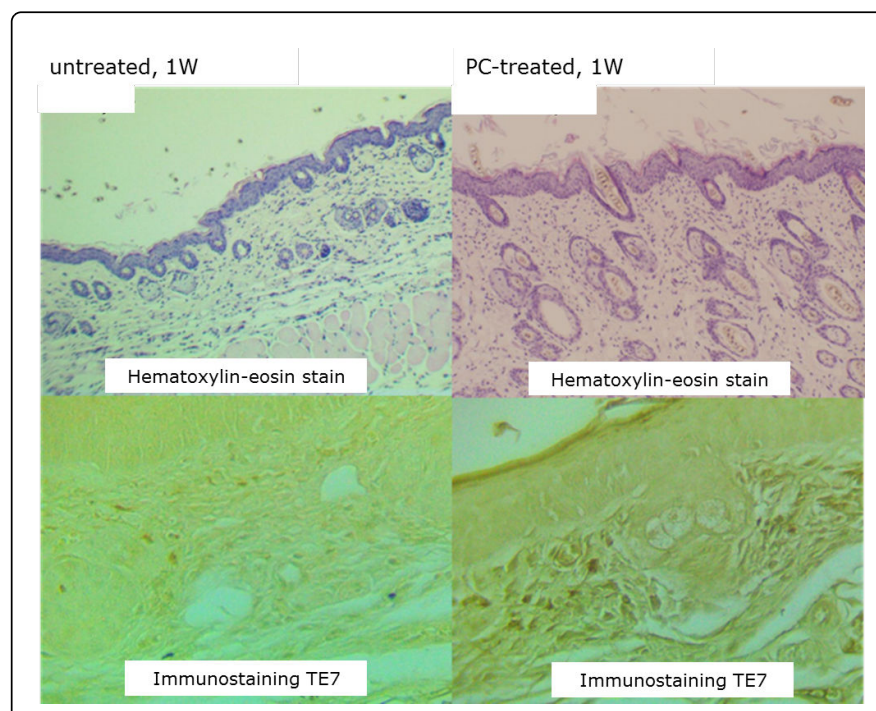

Figure 4: Skin tissue 1 week after $\mathrm{PC}$ administration after hematoxylin-eosin staining (top) and immunostaining (below). Left: untreated; right: PC-treated. A band above each image is epidermis and the blue part is the dermis.

Therefore, it is suggested that PC accelerates wound healing during the acute phase of $\mathrm{AD}$ through promoting the proliferation of fibroblasts. In the future, we plan to investigate how the effect of PC varies depending on the pathology of $\mathrm{AD}$.

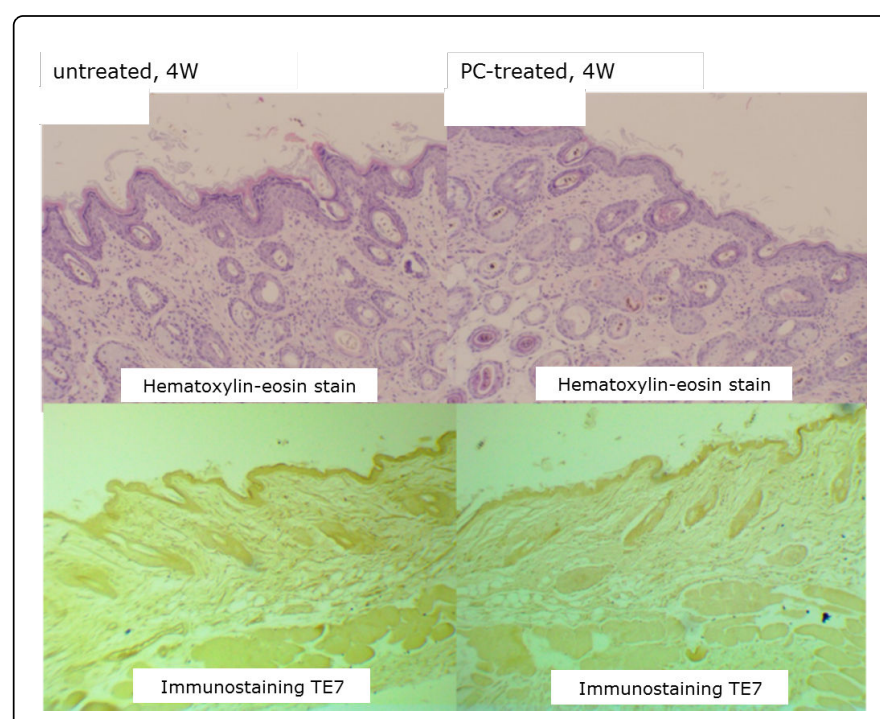

Figure 5: Skin tissue 4 weeks after PC administration after hematoxylin-eosin staining (top) and immunostaining (below). Left: untreated; right: PC treated. A band in the upper part of each image is epidermis and the blue part is the dermis. 
Page 5 of 5

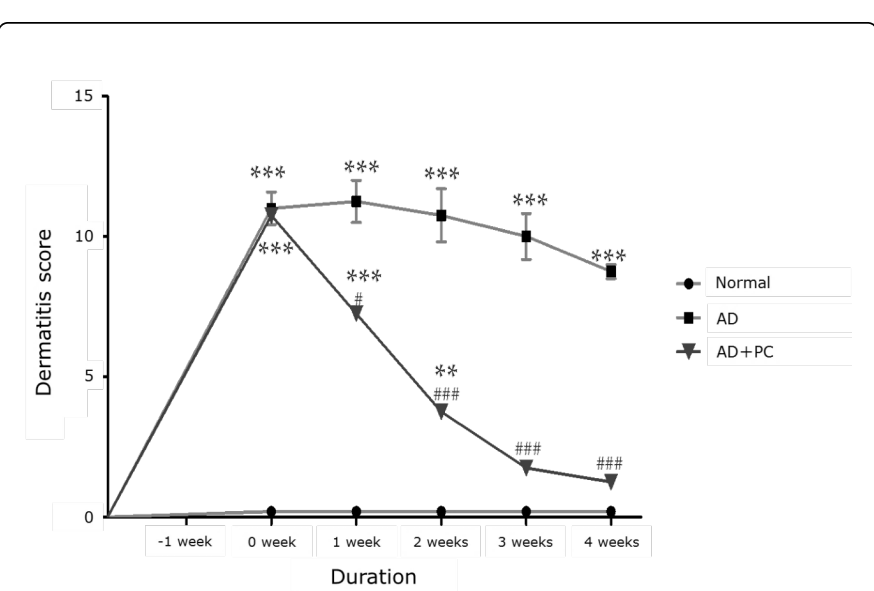

Figure 6: Changes in dermatitis score. ${ }^{*} \mathrm{p}<0.01$ and ${ }^{\star *} \mathrm{p}<0.001$ vs Normal; $\# \mathrm{p}<0.05$ and \#\#\#p<0.001 vs AD.

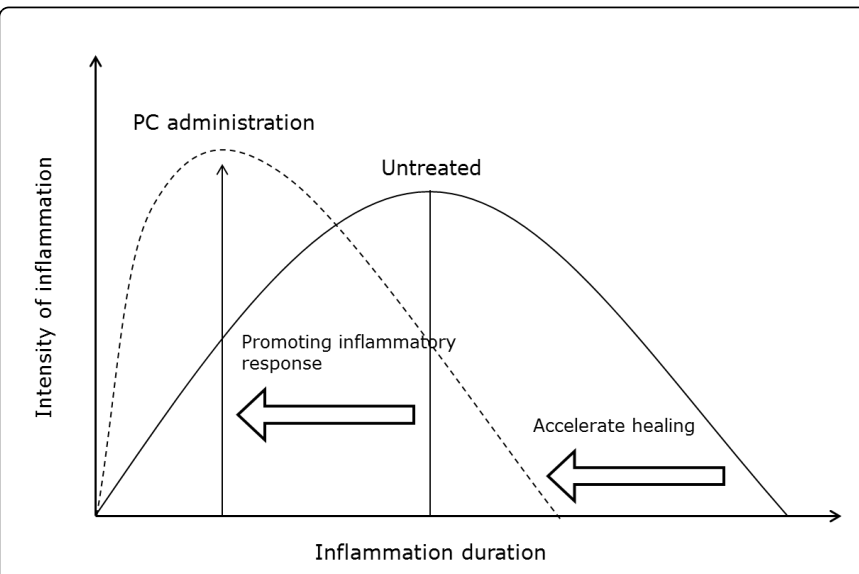

Figure 7: Estimated mechanism of PC. PC promotes the inflammatory response and accelerates healing.

\section{Conflict of Interest}

Dr. Nomoto reports non-financial support (the gift of the PC extract) from Kracie Pharma, Ltd. during the conduct of the study and personal fees (consulting fee/honorarium) from Kracie Pharma, Ltd. outside of the submitted work.

\section{References}

1. Tohno H, Horii C, Fuse T, Okonogi A, Yomoda S (2010) Evaluation of estrogen receptor Beta binding of pruni cortex and its constituents. Yakugaku Zasshi 130: 989-997.

2. Yoshinari K, Shimazaki N, Sashida Y, Mimaki Y (1990) Flavanone xyloside and lignans from Prunus jamasakura bark. Phytochem 29: 1675-1678.
3. Hashiro M, Matsumoto C, Takijiri C, Kitaba S, Murota H, et al. (2011) Effect of Jumihaidokuto (Shi-Wei-Bai-Du-Tang) on dermatological symptoms of patients with atopic dermatitis: evaluation of eruption elements. Skin Res 10: 34-40.

4. Tohno H, Yomoda S, Takemura T (2014) Significance of cherry barkformulation in Jumihaidokuto (Shi-Wei-Bai-Du-Tang) for acne vulgaris treatment. BIO Clinica 3: 124-131.

5. Nakanishi T, Inoue K (2011) Antioxidant potential of Jumihaidokuto. Kampo New Ther 20: 89-91.

6. Shinohara K, Fujita N (2016) The Effect of Jumihaidokuto (Shi-Wei-BaiDu-Tang) and Cherry Bark (Pruni Cortex) on sebum synthesis. Jpn J Med Pharm Sci 73: 579-583.

7. Chiba S, Fujita N, Yomoda S, Takahashi R (2016) Effect of Pruni Cortexcontaining Jumihaidokuto (Shi-Wei-Bai-Du-Tang) on Propionibacterium acnes-induced inflammatory responses of neutrophil. Jpn J Med Pharm Sci 73: 1265-1273.

8. Watanabe K, Karuppagounder V, Arumugam S (2015) Pruni cortex ameliorates skin inflammation possibly through HMGB1-NFkB pathway in house dust mite induced atopic dermatitis NC/Nga transgenic mice. J Clin Biochem Nutr 56: 186-194.

9. Ishihara A, Hashimoto Y, Tanaka C, Dubouzet JG, Nakao T, et al. (2008) The tryptophan pathway is involved in the defense responses of rice against pathogenic infection via serotonin production. Plant J 54: 481-495.

10. Takemoto JK, Remsberg CM, Yáñez JA, Vega-Villa KR, Davies NM (2008) Stereospecific analysis of sakuranetin by high -performance liquid chromatography: pharmacokinetic and botanical applications. J Chromatogr B Analyt Technol Biomed Life Sci 875: 136-141.

11. Kim JR, Choi J, Kim J, Kim H, Kang H, et al. (2014) 20-O- $\beta$-Dglucopyranosyl-20(S)-protopanaxadiol-fortified ginseng extract attenuates the development of atopic dermatitis-like symptoms in NC/Nga mice. J Ethnopharmacol 151: 365-371.

12. Lim HS, Seo CS, Ha H, Lee H, Lee JK, et al. (2012) Effect of Alpinia katsumadai Hayata on House Dust Mite-Induced Atopic Dermatitis in NC/Nga Mice. Evid Based Complement Alternat Med 2012: 705167.

13. Hanifin JM, Thurston M, Omoto M, Cherill R, Tofte SJ, et al. (2001) The eczema area and severity index (EASI): assessment of reliability in atopic dermatitis. Exp Dermatol 10: 11-18.

14. Bauer EA, Stricklin GP, Jeffrey JJ, Eisen AZ (1975) Collagenase production by human skin fibroblasts. Biochem Biophys Res Commun 64: $232-240$.

15. Ohara H, Ichikawa $\mathrm{S}$, Matsumoto H, Akiyama M, Fujimoto N, et al. (2010) Collagen-derived dipeptide, proline-hydroxyproline, stimulates cell proliferation and hyaluronic acid synthesis in cultured human dermal fibroblasts. J Dermatol 37: 330-338.

16. Kishimoto S (2003) Cutaneous wound healing: The mechanism and treatments. Jpn J Dermatol 113: 1087-1093.

17. Katayama I (2002) Atopic dermatitis and remodeling. Clin Dermatol 56: 39-42.

18. Andreas B, Jochen K, Nina K, Agatha S, Franz S, et al. (2013) Role of fibroblasts in the pathogenesis of atopic dermatitis. J Allergy Clin Immunol 131: 1547-1554.

19. Muraoka K, Yoshida S, Hasegawa K, Nakanishi N, Fukuzawa I, et al. (2003) Pharmacological study on the mechanism of action "Kakkon-to" preparation: Body temperature rise and phagocytic activation of macrophages in dogs. J Trad Med 20: 30-37. 